\title{
Valoración de los conocimientos en primeros auxilios en combate que tienen los soldados españoles
}

\author{
Navío Fernández S. ${ }^{1}$, Fúnez Ñacle M. ${ }^{2}$
}

Sanid. mil. 2015; 71 (1): 8-14; ISSN: 1887-8571

\section{RESUMEN}

Los militares en misiones internacionales están expuestos a múltiples riesgos que ponen en peligro sus vidas. Tener conocimientos de primeros auxilios y habilidad en el manejo del herido grave, pone al alcance del soldado las herramientas necesarias para brindar la primera asistencia a un herido. Es imprescindible asegurar en primer lugar el escenario del incidente antes de exponernos o comprometer al resto de la patrulla. Objetivo general: Evaluar el nivel de conocimientos en primeros auxilios que tienen los soldados participantes en el estudio. Objetivos específicos: $1^{\circ}$ - Valorar si los soldados del Batallón creen estar preparados para prestar los primeros auxilios a un compañero herido en combate. $2^{\circ}$ - Analizar si tienen en cuenta la seguridad del escenario donde ocurrió el suceso antes de socorrer a las víctimas. $3^{\circ}$ - Comprobar si son capaces de identificar una hemorragia externa grave y una situación de paro cardiorrespiratorio, y poder brindar los primeros auxilios. Material y método: Estudio cuantitativo, descriptivo de carácter prospectivo y transversal. Sobre una muestra de 269 soldados de los cuales, 178 realizan una encuesta que nos aporta los datos necesarios para evaluar los conocimientos y habilidades en primeros auxilios y valorar la seguridad del escenario. Resultados: Los resultados nos aportan los datos suficientes para valorar el nivel de conocimientos que tienen en primeros auxilios, si disponen de las habilidades necesarias, si creen estar capacitados para socorrer a este tipo de herido y por último si controlan adecuadamente la seguridad del escenario. Conclusiones: Los soldados del Batallón creen disponer de los conocimientos necesarios para realizar el control de la zona de siniestro, de los conocimientos necesarios para brindar los primeros auxilios a un herido en combate y se creen capacitados para ello.

PALABRAS CLAVE: Seguridad en la escena del incidente, Primeros auxilios y torniquete, Herido en combate.

\section{Appreciating knowledge in first aid in combat with the Spanish soldiers}

SUMMARY: In international missions the military are exposed to multiple risks putting their lives in danger. Having first aid knowledge and skills in the treatment of serious injuries, gives soldiers the necessary tools to provide first aid to a wounded person. First of all it is essential to assure the safety of the scene of the incident before exposing ourselves or others to possible danger. General objective: Assess the level of first aid knowledge that the soldiers taking part in the studio have. Specific objectives: 1 . Check if the battalion of soldiers believes they are ready to provide first aid to a partner injured whilst in combat. 2. Analyse if they take into consideration the safety of the incident location before helping the victims. 3. Check if they are trained to identify a serious external haemorrhage and a situation of cardiorespiratory arrest to be able to provide first aid. Material and method: A quantitative study, and prospective and transversal descriptive. With a sample of 269 soldiers, of which 178 of them complete a survey that provides the necessary data to evaluate first aid knowledge and abilities and the checking the safety of the scene. Results: The results provide sufficient information to evaluate the soldiers' level of first aid knowledge, whether or not they have the necessary skills, if they believe that they are capable of dealing with these kinds of wounds, and if they control correctly the security of the scene. Conclusions: The battalion of soldiers believe they have all the necessary knowledge to correctly control the scene of the accident, the necessary knowledge to provide first aid to soldier wounded in combat, and they believe are capable providing first aid.

KEYWORDS: Assuring the scene of the incident, First aid and tourniquet, Wounded in combat.

\section{INTRODUCCIÓN}

El shock hipovolémico por hemorragia aguda y el Paro Cardio Respiratorio (PCR), son dos de las situaciones más graves que puede presentar un soldado herido durante el combate ${ }^{1}$.

Definimos el shock hipovolémico como un estado de hipoperfusión tisular provocado por una hemorragia exanguinante

\footnotetext{
${ }^{1}$ Cap. Enfermero. Comandancia de la Guardia Civil de Barcelona. España.

${ }^{2}$ Cap. Enfermero. BCZM Barcelona IV/62. Barcelona. España.

Dirección para correspondencia: snaviof@guardiacivil.es; mfunnac@et.mde.es

Recibido: 7 de febrero de 2014

Aceptado: 5 de septiembre de 2014
}

con la consiguiente disminución del aporte de oxígeno a órganos y tejidos ${ }^{1}$. El sistema nervioso central es especialmente sensible a la hipoxia, pudiendo provocar lesiones irreversibles a nivel cerebral e incluso la muerte del paciente.

Una hemorragia externa exanguinante originada por un trauma vascular grave, ocasiona una pérdida hemática importante que originará un deterioro hemodinámico progresivo que evolucionará a shock hipovolémico y que si no se resuelve, provocará la muerte del paciente ${ }^{2}$.

Por último el PCR es el cese súbito de la respiración y de las contracciones del corazón. Es una situación clínica crítica, que por razones patológicas intrínsecas o por una agresión externa, en la que se produce un cese de los movimientos respiratorios y por lo tanto del intercambio de gases respiratorios (oxígeno y 
dióxido de carbono o $\mathrm{CO}_{2}$ ) a nivel de los alveolos pulmonares 3 . Asociado a este fracaso respiratorio, sumamos el cese de las contracciones cardíacas, responsables estas de la distribución de la sangre con el oxígeno y con todos los elementos necesarios para el metabolismo celular

Ambas situaciones son potencialmente reversibles; en la hemorragia, si disponemos de los conocimientos básicos necesarios para identificar los signos que definen la gravedad de la situación clínica y si contamos con la destreza suficiente para realizar de forma eficaz las técnicas terapéuticas urgentes dirigidas a cohibir esta hemorragia, evitaremos la fatal evolución al cuadro de shock hipovolémico ${ }^{3}$.

Del mismo modo, en el caso del PCR, la aplicación de forma precoz y eficaz de las maniobras de resucitación o reanimación cardiopulmonar, representa la única opción terapéutica eficaz para salvar esta crítica situación clínica ${ }^{4}$.

\section{Antecedentes y situación actual}

Del instinto destructor derivado de los conflictos bélicos, surge la necesidad de crear una serie de elementos de protección al servicio de los combatientes (cascos, escudos, armaduras, blindajes de vehículos, refugios, etc.) y desarrollar técnicas de control y tratamiento de lesiones propias del combate ${ }^{5}$. Las regiones anatómicas donde se registran lesiones vasculares más graves son el cuello y la región inguinal. Un traumatismo penetrante localizado en estas zonas conlleva, en la mayoría de los casos, la afectación de grandes vasos con una grave repercusión hemodinámica e incluso pudiendo causar la muerte del combatiente en pocos minutos. La gran movilidad que necesitan las tropas hace que estas regiones anatómicas sean difíciles de proteger en comparación con la cabeza y tórax, que sí cuentan con evolucionados sistemas de protección.

Cuando estos dispositivos no son eficaces la única posibilidad de salvar la vida del soldado herido es la rápida valoración de las lesiones sufridas y su tratamiento de forma inmediata.

No olvidemos la importancia que tiene en este contexto la «seguridad del escenario». Se debe tener presente, que en el combate la seguridad de la zona donde se ha producido la agresión es tanto o más importante que la propia asistencia a la baja ${ }^{2}$. Una vez asegurada la zona del incidente y tratadas las lesiones del paciente se puede realizar su evacuación al puesto quirúrgico avanzado o al hospital de campaña, donde recibirá una asistencia médica o quirúrgica más especializada. Es en la primera línea de combate donde se producen el mayor número de muertes «potencialmente evitables» y donde un precoz y eficaz tratamiento in situ de las lesiones salvará un elevado número de vidas ${ }^{6}$.

Entre las responsabilidades de la Sanidad Militar de los Ejércitos se encuentra la de velar por la salud de los soldados desplazados a zona de operaciones. Una adecuada formación y un entrenamiento específico garantizarán una rápida protección del lugar del incidente, una eficiente valoración y una eficaz asistencia inicial de las lesiones derivadas del combate ${ }^{7,8}$.

Para conseguir la excelencia en el desarrollo de sus competencias profesionales en estos difíciles escenarios, deberá llevarse a cabo un completo y continuado adiestramiento en las técnicas de asistencia prehospitalaria como base para el tratamiento ini- cial y puesta en estado de evacuación de las bajas en este contexto $^{9-11}$. Para cerrar el complejo círculo de la asistencia urgente al herido grave en el combate, nos centraremos en los conocimientos que tiene el personal sanitario de tropa integrado en la unidad combatiente y en la instrucción que recibe el soldado en materia de primeros auxilios. Esta formación constituye uno de los pilares en los que se sustentará el éxito de la asistencia inicial de la baja de combate.

El personal sanitario de las secciones o pelotones y el propio combatiente, son los responsables de brindar la primera asistencia a la baja de combate, disminuyendo con una adecuada actuación, las denominadas muertes evitables en combate. Esta mortalidad puede reducirse mediante la realización de sencillas medidas salvadoras de la vida en primera línea. En la actualidad la formación en materia de primeros auxilios para personal de Tropa y Marinería se realiza por dos vías diferentes pero orientadas al mismo objetivo.

$1^{\circ}$ - Todo soldado que ingresa en un centro docente de formación militar para tropa y marinería, recibe la formación militar básica y la específica de su especialidad. Según el actual plan de formación sanitaria del ET la clasificación de dichos cursos es la siguiente:

- Cursos de Primeros Auxilios (Código FSET 1) son un total de 20 horas lectivas, distribuidas en 13 tareas de nivel I que están desarrolladas en el M17-021. Cada tarea tiene una duración de 90 minutos, la mayoría con 1/3 dedicado a teoría y $2 / 3$ a práctica. Se impartirá en los Centros de Formación y Academias durante la Fase General Militar.

- Curso de Instructor de Primeros Auxilios (Código FSET 2) su finalidad es formar a los Cuadros de Mando y Tropa que se determine para poder ejercer las tareas de instructor en los cursos FSET 1 que se realicen en sus Unidades o centros bajo dirección de personal facultativo. Incluirá las tareas de nivel I sobre Primeros Auxilios y un módulo sobre técnicas de instrucción, simuladores de incidencias y preparación y mantenimiento del material necesario para cada una de las sesiones prácticas. Deberá reunir los requisitos necesarios para su homologación por la SEMICYUC.

- Curso de Sanitario (Código FSET 3) antigua titulación de técnico en emergencias avanzado, actualmente descatalogada. Se imparte desde el año 2007 en la Escuela de Sanidad con una duración de 185 horas teórico prácticas de las cuales 85 corresponden a prácticas en ambulancias de los servicios de emergencias de la CCAA de Madrid.

$2^{\circ}$ - La importancia de la formación continuada en las unidades de destino corresponde al personal facultativo de la Sanidad Militar, dentro de su programa anual de formación, el impartir los cursos básicos de primeros auxilios y conferencias sanitarias de interés general para la tropa ${ }^{1,10}$.

Como preparación más avanzada del personal de Tropa Profesional que actuará como sanitario y antes de desplazarse las unidades a zona de operaciones en el extranjero, la DISAN del ET dentro de programas propio de formación e instrucción para estos profesionales, pueden realizar el curso Tactical Combat Casuality Care (TCCC) ${ }^{12}$, formación específica orientadas a resolver eficazmente situaciones similares en las que el soldado puede encontrarse ${ }^{12,13}$. 
El curso Prehospital Trauma Life Support (PHTLS) ${ }^{9}$ y el PHTLS edición militar ${ }^{14}$ son cursos dirigidos a personal de Medicina y Enfermería dentro del plan de formación continuada propia de la DISAN del ET.

El estudio PROCES (Programa de Reanimación Cardiopulmonar Orientado a centros de Enseñanza Secundaria) $)^{15}$, demuestra con una experiencia adquirida tras 5 años de formación en SVB, que el porcentaje de aprendizaje satisfactorio inmediato y tras un periodo de tiempo es significativo. Buscar analogías en procedimientos o contenidos, adoptar otros métodos o programas en nuestro sistema de enseñanza, nos puede ayudar a conseguir la excelencia en la formación del soldado.

\section{Justificación}

\section{Pertinencia}

La pertinencia de este estudio radica en que gran parte de las causas potenciales de muerte durante acciones de combate o bien, durante realización de ejercicios de instrucción y adiestramiento de alto riesgo de nuestras tropas pueden ser evitables mediantes el conocimiento de sencillas maniobras salvadoras de la vida que pueden permitir aumentar el tiempo de supervivencia de los combatientes hasta la llegada de los servicios médicos y de personal especializado.

\section{Relevancia}

Una preocupación de todos los Ejércitos a lo largo del siglo XX y siglo XXI ha sido aumentar la supervivencia de los combatientes en los conflictos bélicos. Contamos para ello con herramientas que favorecen el aumento de dicha supervivencia como son una adecuada formación en materia de primeros auxilios que permita identificar y brindar una asistencia inicial con el fin de recuperar el mayor número de soldados heridos.

\section{OBJETIVOS}

\section{Objetivo general}

Evaluar el nivel de conocimientos en primeros auxilios que tienen los soldados de un batallón de cazadores de montaña.

\section{Objetivos específicos}

$1^{\circ}$ - Valorar si los soldados del Batallón de Cazadores de Montaña «Barcelona IV/62» creen estar preparados para prestar los primeros auxilios a un compañero herido en combate.

$2^{\circ}$ - Analizar si se tiene en cuenta la seguridad del escenario antes de socorrer a las víctimas.

$3^{\circ}$ - Comprobar el grado de conocimientos a la hora de identificar una hemorragia externa grave, una situación de P.C.R. y de aplicar los primeros auxilios a un herido en dichas situaciones.

\section{MATERIAL Y MÉTODO}

\section{Tipo de estudio}

Se trata de un estudio cuantitativo, descriptivo, de carácter prospectivo y transversal.

\section{Población}

La muestra del estudio está compuesta por 354 soldados de los que únicamente 269 cumplían los criterios de inclusión y de los cuales 178 disponibles en ese momento en la Unidad realizaron la encuesta, distribuidas entre los empleos de cabos $1^{\circ}$, cabos y soldados, todos destinados en el Batallón de Cazadores de Montaña Barcelona IV / 62. Este batallón es una unidad operativa perteneciente a las Fuerzas Terrestres del Ejército de Tierra, especializada en el movimiento y el combate en montaña y alta montaña.

\section{Criterios de inclusión}

Cabos $1^{\circ}$, cabos y soldados en activo, destinados en el Batallón de Cazadores de Montaña Barcelona IV / 62, y que no tienen ninguna limitación para realizar las actividades propias de su unidad y especialidad, estas son:

- $1^{\mathrm{a}}$ y $2^{\mathrm{a}}$ compañía de Cazadores (fusileros).

- Compañía de Apoyo (sección de armas de apoyo y sección de reconocimiento).

- Compañía de Servicios.

\section{Criterios de exclusión}

Cabos $1^{\circ}$, cabos y soldados que se encuentran en alguna de las siguientes situaciones:

- Incapacidad temporal para el servicio por enfermedad o accidente.

- Personal en excedencia por maternidad, asuntos propios o en comisiones de servicio.

- Cabos $1^{\circ}$, cabos y soldados destinados en las Planas Mayores de Mando, por no estar estos integrados en la unidad como soldados combatientes.

- El personal destinado en la Sección de Sanidad del batallón, por estar especialmente instruido en la asistencia sanitaria del combatiente en primera línea de fuego.

- Los Oficiales y suboficiales del batallón, por tener misiones específicas de dirección y control de acciones propias del combate.

\section{MATERIAL Y MÉTODO}

Los datos se recogieron a través de una encuesta del tipo auto administrada y anónima. En su inicio se preguntan datos de filiación de interés excluyendo los de identificación (nombre y DNI), seguido de datos alusivos al nivel académico y de conoci- 
mientos específicos en primeros auxilios o formación sanitaria, diferenciando 2 niveles (superior o inferior a 20 horas lectivas). En la parte final se interroga al encuestado si «cree estar preparado para atender a un herido grave en combate», pregunta relacionada con uno de los objetivos del estudio.

En la segunda parte de la encuesta se formulan 10 preguntas de evaluación de los conocimientos en primeros auxilios de los encuestados. Entre ellas existen preguntas claves encaminadas a identificar tres aspectos relevantes del estudio. Son:

$1^{\circ}$ - Pregunta $n^{\circ} 4$. «Seguridad del escenario», la capacidad para controlar la seguridad del escenario donde sucedió el incidente, considerado como punto clave e inexcusable en una adecuada intervención en combate o en cualquier otra situación de riesgo con víctimas.

$2^{\circ}$ - Pregunta $n^{\circ} 1$ y 5 . «Identificación e inmediato tratamiento de la hemorragia externa exanguinante» la intervención que más vidas puede salvar entre los combatientes.

$3^{\circ}$ - Preguntas $n^{\circ}$ 6, 7 y 8. «Identificación e inmediata asistencia inicial del Paro Cardíaco, Respiratorio o ambos simultáneamente».

El resto de las preguntas simplemente aportan información del nivel general de conocimientos en primeros auxilios.

La recogida de datos se realizó por compañías los días 21, 22 y 27 de noviembre del año 2013, sólo a los soldados que se encontraban disponibles en ese momento en la unidad y que cumplían los criterios de inclusión en el estudio. Se utilizó la misma encuesta para todos los participantes, se entregó en mano junto con el guion informativo y el consentimiento, informándose del procedimiento del estudio realizando las aclaraciones oportunas y resolviendo las dudas.

Las variables estudiadas son del tipo cualitativas y los resultados expresados en porcentajes y frecuencia.

Para la corrección del apartado de la encuesta que va orientada a la valoración de conocimientos teóricos, se ha utilizado el sistema de corrección del Consejo Catalán de Resucitación, que establece un $70 \%$ de respuestas correctas para acreditar que se tiene los conocimientos suficientes en la materia, criterio que se ha extrapolado al resto del cuestionario.

\section{Análisis de datos}

Para el análisis de los datos se utiliza estadística descriptiva usando estadísticos de tendencia central como: media, mediana, moda y medidas de dispersión como la desviación típica.

La base de datos ha sido elaborada mediante la utilización del programa informático IBM - SPSS 14.0, y base de datos Excel.

\section{Aspectos éticos}

Para realizar el presente estudio se ha solicitado la autorización del Coronel jefe de Regimiento Cazadores de Montaña Arapiles 62 y al jefe del Batallón de Cazadores de Montaña Barcelona IV/62.

Se solicita la autorización expresa de cada uno de los participantes en el estudio.
De conformidad con lo previsto en la Ley Orgánica 15/1999 de 13 de diciembre, de Protección de Datos de Carácter Personal (BOE num. 298 de 14 de diciembre de 1998), toda la información de carácter personal contenida en este estudio estarán sometidos al amparo de dicha ley.

\section{RESULTADOS}

Población del estudio ( $\mathrm{n}=269)$. Número de encuestas entregadas 183 unidades, en estas se excluyen 5 que son realizadas a modo de control por personal facultativo y sanitario de la Unidad, incorporándose finalmente al estudio 178 encuestas. Representa una tasa de respuestas del 66.17\% $(n=178)$.

En la variable edad resulta una población relativamente joven, observamos unos límites inferior y superior comprendidos entre los 18 y 37 años respectivamente con los siguientes resultados: Media $=25.5$ años, mediana $=25.0$ años, moda $=24.0$ años y la desviación típica de $=3.08$ años.

Con relación al Sexo obtenemos un marcado predominio de los varones frente a las mujeres, del total de encuestados (178) contamos con un $94.9 \%(n=169)$ de varones y $5.1 \%(n=9)$ de mujeres (Figura 1).

Los valores relacionados con el nivel académico general de los participantes son los siguientes resultados: Estudios Primarios $(\mathrm{P}) 5.1 \%(\mathrm{n}=9)$, Enseñanza Superior Obligatoria (ESO) 59.6\% $(\mathrm{n}=106)$, Bachiller $(\mathrm{B}) 27.5 \%(\mathrm{n}=49)$, Técnico Superior (TS) $5.6 \%(\mathrm{n}=10)$ y Licenciados o Diplomados (ES) $2.2 \%(\mathrm{n}=$ 4) (Figura 2).

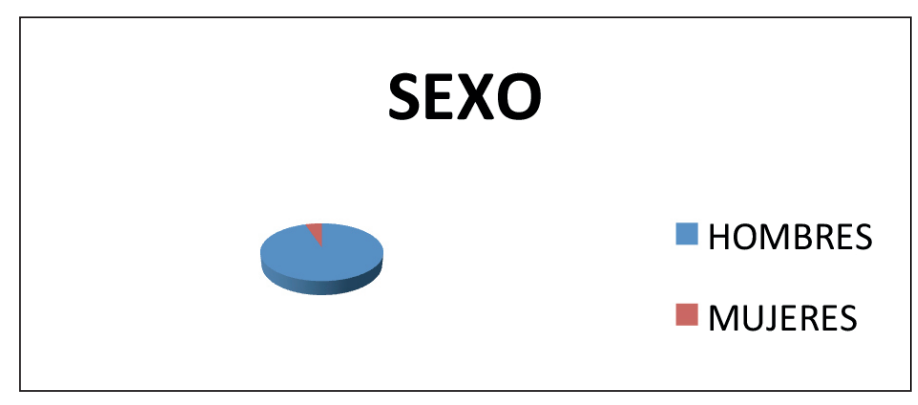

Figura 1.

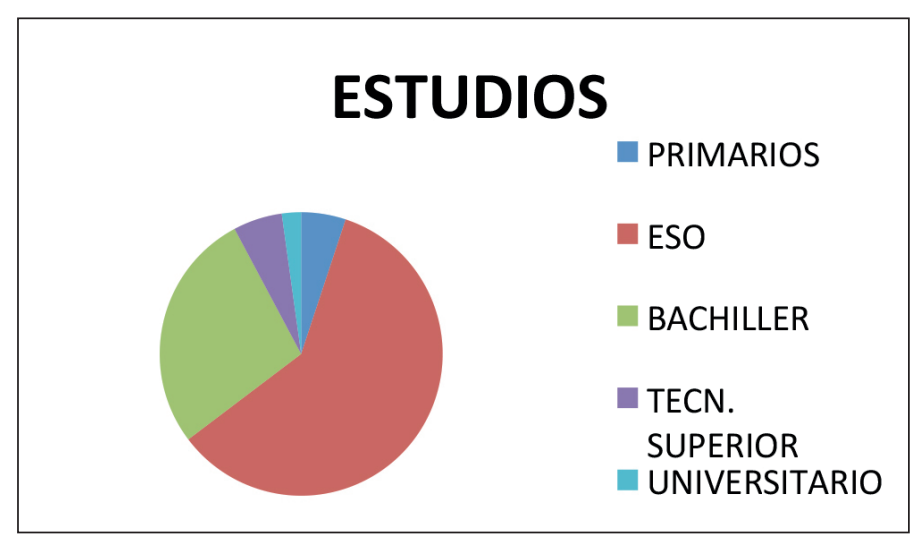

Figura 2. 
Relacionado con el nivel académico pero especificando en los conocimientos en Primeros Auxilios, diferenciamos dos grupos, los que sí han realizado algún curso de socorrismo y los que no. De ser afirmativa la respuesta, diferenciaremos los que han recibido curso superior o inferior a 20 horas lectivas. Los resultados son los siguientes: Conocimiento en primeros auxilios No el 70.2\% (n $=125)$, Sí el 29.8\% $(\mathrm{n}=53)$, de estos 53 soldados que sí cuentan con estos conocimientos, son con curso $<20$ horas el 26.4\% $(\mathrm{n}=$ 14) y con curso $>20$ horas el $73.6 \%(n=38)$ (Figura 3$)$.

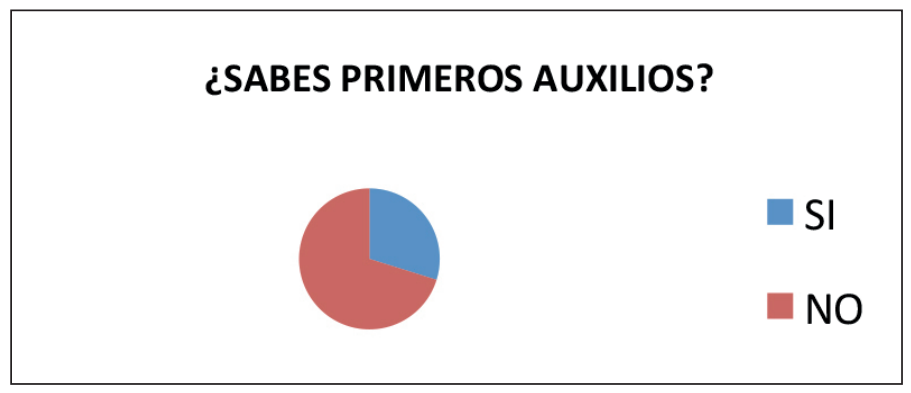

Figura 3.

Una de las preguntas claves del cuestionario es «cree estar preparado para prestar los primeros auxilios a un compañero herido grave en combate», contestan Sí $=109(61.3 \%)$ y No $=69$ (38.7\%) (Figura 4).

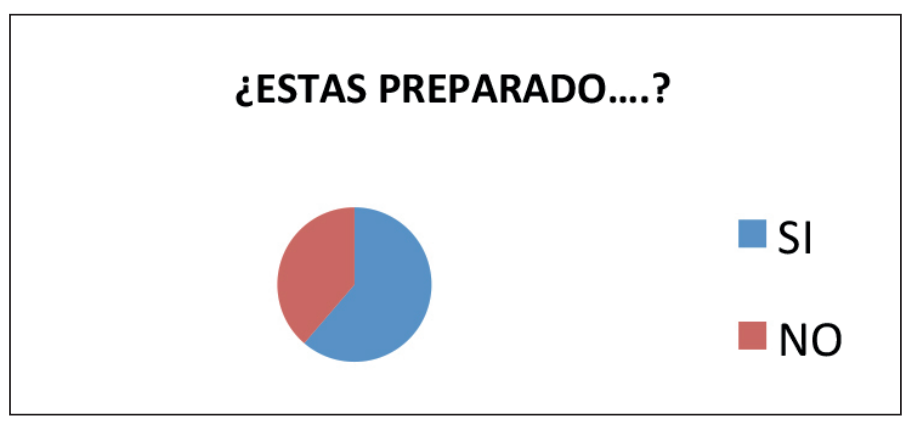

Figura 4

Con relación a la «notas» obtenidas en el cuestionario y tomando como referencia las recomendaciones del Consejo Catalán de Resucitación, solicitamos de los encuestados superar como mínimo del 70\% de respuestas correctas para considerar que cuentan con los conocimientos suficientes en técnica de reanimación y aplicados igualmente los de primeros auxilios. Los resultados estadísticos de los 178 encuestados son los siguientes:

Igualan o superan el 70\% de aciertos. Sí = 169 (94.3\%), No $=9(5.7 \%)$, media $=8.67$, mediana $=9.000$, moda $=9.0$ y desviación típica $=1.37$. Con relación a la frecuencia con que se repiten cada uno de los valores de las notas, son: Preguntas acertadas (pa.), 2 pa. el $0.6 \%(\mathrm{n}=1), 3$ pa. el $0.6 \%(\mathrm{n}=1), 4$ pa. el $1.1 \%(n=2), 6$ pa. el $3.4 \%(n=6), 7$ pa. el $10.1 \%(n=18), 8$ pa. el $20.8 \%(n=37), 9$ pa. el $32 \%(n=57)$ y 10 pa. el $31.5 \%(n=56)$ (Figura 5).

Por último intentamos saber si los soldados encuestados están capacitados para valorar y responder adecuadamente tres
VALORARON LA SEGURIDAD DEL ESCENARIO

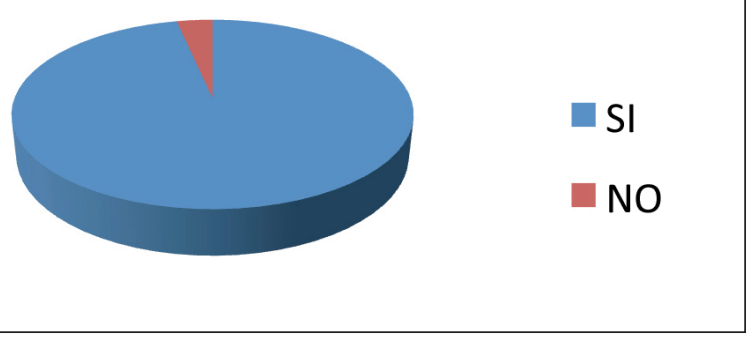

Figura 6.

\section{IDENTIFICAN Y CONOCEN LOS PROCEDIMIENTOS DE ATENCIÓN HEMORRAGIA GRAVE EN COMBATE}

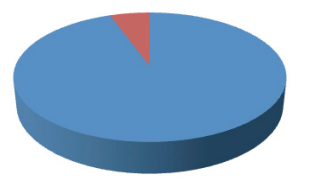

\section{IDENTIFICAN UNA PARADA RESPIRATORIA I/O CARDIACO Y REALIZAN TRATAMIENTO}

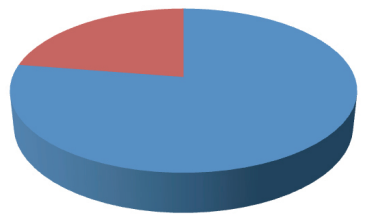

SI

NO

Figura 8.

situaciones que comprometen gravemente la vida del herido o de los integrantes de la patrulla, estas situaciones son:

$1^{\circ}$ - Relacionado con la seguridad del escenario. «Son los soldados capaces de anteponer la seguridad del escenario a cualquier actuación sanitaria». Contestaron que sí valoraron previamente el escenario el $96.6 \%(n=172)$ y no valoraron el escenario antes de intervenir el 3.40\% $(n=6)$ (Figura 6).

$2^{\circ}$ - Con relación a la identificación y asistencia inicial de una hemorragia externan grave. Saben identifican y conocen los procedimientos a aplicar ante una hemorragia externa grave en combate» el 94.4\% $(\mathrm{n}=168)$, no lo hacen el 5.6\% $(\mathrm{n}=10)($ Figura 7$)$.

$3^{\circ}$ - «Identifican la situación de paro respiratorio o cardíaco y conocen los procedimientos de reanimación». Si lo identifican y tratan el $77.5 \%(n=138)$, no lo identifican el $22.5 \%(n=40)$ (Figura 8). 


\section{DISCUSION}

La participación en el estudio representa un $66.17 \%$ de la Unidad estudiada.

Para la puntuación global en las preguntas de conocimientos teóricos en primeros auxilios se siguen las orientaciones del Consejo Catalán de Resucitación. Alcanzaron la calificación de aptos el 94.3\% (n=168) (figura 5), lo que indica un alto números de aprobados. Si comparamos estos resultados con relación a las variables de haber realizado cursos de socorrismo (figura 3) y las del nivel académico o de estudios realizados (figura 2), no le damos tanto valor a estas variables y sí a la concienciación de la importancia de adquirir estos conocimientos.

La primera pregunta clave «cree estar preparado para prestar los primeros auxilios a un compañero herido grave en combate» han contestado «Sí» el 61.3\% (n=109) (figura 4). Esto demuestra que un elevado número de soldados creen tener suficientes conocimientos de socorrismo, para atender adecuadamente a un compañero herido en esas circunstancias.

Con relación a las preguntas claves del cuestionario donde se identifican situaciones o se preguntan tratamientos, las contestan adecuadamente un elevado número de encuestados, estas son:

- La pregunta $n^{\circ} 4$, relacionada con la «seguridad del escenario del suceso», la contestan correctamente el $96.6 \%$ $(n=172)$ lo que demuestra que la mayoría de los soldados si tienen presentes las medidas de seguridad imprescindibles en estas situaciones.

- Las preguntas 1 y 5 versan de la identificación y tratamiento de la hemorragia severa, la superan holgadamente el $94.4 \%(n=168)$ de los encuestados. Esto nos indica que identifican esta situación de extrema gravedad y conocen las medidas a tomar para el control de la hemorragia externa.

- Las preguntas 6, 7 y 8 van orientadas a la identificación y conocimientos de las técnicas de Reanimación Cardio Pulmonar. Un alto porcentaje de encuestados 75.5\% $(n=138)$ las contestan correctamente. Esto aporta al estudio que un elevado número de soldados reconocen e identifican los signos PCR y las medidas de reanimación.

Con relación a la variable sexo, predomina en la población encuestada los varones en un porcentaje del 94.9\%. Este resultado aporta poco interés para el estudio.

Ocurre lo mismo con la edad, no nos sorprende ver que la media de edad de los soldados del batallón es de 25.5 años. El tipo de actividad que realizan requiere una instrucción, un entrenamiento y una buena forma física, lo que lógicamente pone límites a la edad.

Encontramos en el artículo titulado «Control de la hemorragia en el entorno militar $»^{6}$ que hace referencia a los $« 5$ minutos de platino» en clara alusión a la tradicional y deseada «hora de oro» en medicina prehospitalaria. En el combate, la seguridad del escenario donde sucede el incidente, junto al rápido y eficaz tratamiento de las víctimas en el instante inmediato al suceso («cinco minutos de platino») favorecerá, sin duda, el pronóstico de los heridos.

Observamos los resultados obtenidos con otro estudio realizado a la población civil, concretamente en 15 centros educativos de secundaria en la ciudad de Barcelona. Se trata del estudio
«PROCES», que describe la experiencia adquirida tras 5 años de realizar formación en SVB a los alumnos de enseñanza secundaria obligatoria, obteniéndose un porcentaje de aprendizaje satisfactorio inmediato a la formación del 58\%, y una persistencia del mismo, al cabo de un año, se traducía en un $42 \%$. Estos resultados al igual que los obtenidos en la formación de los soldados, demuestran la importancia de incluir la formación inicial en los ciclos educativos de escuelas y academias, así como, el mantener una formación continuada durante la vida laboral de estos.

Refuerza los resultados del estudio el saber que en los Centros de Formación Militar, los soldados reciben unos conocimientos básicos en primeros auxilios. A posteriori en las unidades, se refuerzan con nuevos cursos y conferencias sanitarias. Existe creciente preocupación por parte de los mandos militares en la diversidad y la calidad de la formación del soldado ${ }^{3}$. Han de reforzarse los conocimientos y habilidades en materia de primeros auxilios, durante la fase de concentración previa a un despliegue en $\mathrm{ZO}^{1,12,14}$.

\section{Fortalezas del estudio}

La fortaleza del estudio se basa en el número y la homogeneidad de la muestra, los criterios utilizados para la exclusión de participantes en el estudio y la redacción de la encuesta bajo el asesoramiento de personal cualificado.

\section{Limitaciones del estudio}

La principal limitación del presente estudio se halla en la valoración solo de conocimientos teóricos, es evidente que una buena base teórica es esencial para el adecuado manejo del herido grave y el PCR, pero la destreza práctica, es obviamente un factor determinante a la hora de valorar una correcta asistencia a un herido grave.

No contar con un grupo control en los que todos sus componentes posean los mismos conocimientos en SVB y Primeros Auxilios. Realizar una posterior evaluación pasado un período determinado de tiempo (6 o 12 meses) desde la valoración primaria.

\section{CONCLUSIONES}

1- El nivel de conocimientos en primeros auxilios de los soldados del Batallón de Cazadores de Montaña Barcelona IV/62 es alto, tomando como referencia superar el $70 \%$ de la prueba de conocimientos que aconseja la Sociedad Catalana de Reanimación.

2- La muestra encuestada sí cree estar preparada para presta los primeros auxilios a un compañero herido grave en combate.

3- La muestra encuestada sí es conscientes de la importancia de la seguridad del escenario donde se encuentran las víctimas.

4- La muestra encuestada tiene los conocimientos necesarios para identificar una hemorragia externa grave y una situación de PCR así como aplicar las medidas en materia de primeros auxilios necesarias. 


\section{S. Navío Fernández y M. Fúnez Nacle}

\section{BIBLIOGRAFÍA}

1. Baz Simal A., Benedet Caraballo JE., Cabrera Macías C., Campillo Laguna JR., Cantero Martí JL. FSET-3, Curso de Sanitario en Operaciones. Madrid: Ministerio de Defensa, publicación militar del Ejército de Tierra; 2011.

2. Mando de Adiestramiento y Doctrina. Instrucción Sanitara del Combatiente. Madrid: Ministerio de defensa, publicación militar del Ejército de Tierra; 2012.

3. Mando de Adiestramiento y Doctrina. Manual de Instrucción del Sanitario de la Pequeña unidad. Madrid: Ministerio de Defensa, publicación militar del Ejército de Tierra; 2008.

4. American Heart Associations. Guidelines CPR, ECC 2010. Dallas: American Heart Associations; Nov.2010.

5. Navarro Suay R., Pérez Ferrer A., Jiménez Vizuete JM. Control de la hemorragia en el entorno militar. Rev Esp Anestesiol Reanim 2012 Dec; 59 (10):562-72.

6. Pannell D, Brisebois R, Talbot M, Trottier V, Clement J. Garraway N, et al. Causes of death in Canadian Forces members deployed to Afghanistan and implications on tactical combat casualty care provision. J. Trauma 2011 nov; 71(5 Suppl 1): S401-7.
7. Mando de Adiestramiento y Doctrina. PD4-616, Sanidad en Operaciones. Madrid: Ministerio de defensa, publicación militar del Ejército de Tierra; 2011.

8. Kragh JF.Jr., Swan KG, Smith DC, Mabry RL, Backbourne LH. Los nuevos conceptos de dispositivos de torniquete para el control de la hemorragia en el campo de batalla. Ejercito de los EEUU Med. Dep. J. 2011 apr-jun: 38-48.

9. McSwain NE., Salomone JP., Pons PT. PHTLS, Prehospital Trauma Life Support. $7^{\mathrm{a}}$ edición. Barcelona: Elsevier España S.L.; 2012.

10. Peter T. Pons, MD, Will Chapeau. Técnico en Emergencias Sanitarias. Barcelona: Elsevier; 2008

11. Lee C., Porter KM. Uso del torniquete en el ámbito prehospitalario civil. Emergency Medicine Journal. Agosto 2007; 24 (8): 584-587.

12. Giebner SD., McSwain NE., Jr. Salomone JP. Pons PT. Tactical Combat Casualty Care, PHTLS ed. Military. Barcelona: Elsevier España S.L.; 2011.

13. Maimir Jané F. Asistencia inicial a la baja de combate. Madrid: Unidad Cuidados Intensivos Hospital Central de la Defensa; 2009.

14. Journal of Special Operations Medicine. Lessons learned in Iraq and Afghanistan. Journal of Special Operations Medicine Volumlee 10, Edition 3 / Summer 10.

15. Miró O., Escalada X., Jiménez-Fábrega X., Díaz N., Sanclemente G., Gómez X., et al. Programa de Reanimación Cardiopulmonar Orientado a Centros de Enseñanza Secundaria (PROCES): Conclusiones 5 años de experiencia. Barcelona: Emergencias 2008; 20:229-236. 